\title{
TUBERCULOSIS
}

\section{The growing impact of HIV infection on the epidemiology of tuberculosis in England and Wales: 1999-2003}

\author{
Aliko B Ahmed, Ibrahim Abubakar, Valerie Delpech, Marc Lipman, Delia Boccia, Josh Forde, \\ Delphine Antoine, John M Watson
}

See end of article for authors' affiliations

Correspondence to: Dr John M Watson Respiratory Diseases Department, Centre for Infections, Health Protection Agency, London NW9 5EQ, UK; john.watson@hpa.org. uk

Received 29 September 2006 Accepted 12 January 2007 Published Online First 20 February 2007

\begin{abstract}
Background: Previous studies have estimated the prevalence of tuberculosis and HIV infection in population subgroups in the UK. This study was undertaken to describe recent trends in the proportion of individuals with HIV infection among reported cases of tuberculosis in England and Wales, and to review the implications for clinical and public health care.

Methods: A population-based matching study using national surveillance databases was used to investigate all persons aged 15 years and over reported with a diagnosis of tuberculosis to the Health Protection Agency in England and Wales in 1999-2003. Record linkage was used to match the national tuberculosis and HIV/ AIDS surveillance databases to identify all cases of tuberculosis and determine the proportion of patients with tuberculosis co-infected with HIV. The distribution and characteristics of the cases were determined and the trend examined by year.

Results: Of 30670 cases of tuberculosis reported in England and Wales between 1999 and 2003, an estimated $1743(5.7 \%)$ were co-infected with HIV. There was a year on year increase in the proportion from $3.1 \%(169 / 5388)$ in 1999 to $8.3 \%(548 / 6584)$ in 2003 (p for trend $<0.0001)$. Co-infected patients contributed to almost a third of the increase in the number of cases of tuberculosis during the 5 year period. Patients co-infected with HIV were predominantly those born abroad. 18.5\% ( $n=323)$ of co-infected patients had not been reported as active cases of tuberculosis on the national tuberculosis database.

Conclusion: The proportion of patients with tuberculosis co-infected with HIV in England and Wales is increasing, with the greatest impact on those born abroad regardless of their ethnic origin. With HIV infection contributing substantially to the increase in the number of cases of tuberculosis, close cooperation in the clinical management and accurate notification of patients is vital if appropriate care and public health action is to be achieved.
\end{abstract}

$\mathrm{T}$ he increase in tuberculosis in the UK since the 1980s has been largely attributed to the fact that more people who originate from countries with a high prevalence of tuberculosis subsequently develop tuberculosis while resident here. ${ }^{1}$ Co-infection with HIV is also believed to have contributed, especially in those from countries where the prevalence of HIV infection is high. ${ }^{2}$

The association between tuberculosis and HIV is of great public health importance. HIV infection increases the risk of progression from latent to active tuberculosis and, if untreated, these individuals are more likely to progress to severe disease and die either from tuberculosis or other comorbidities. ${ }^{3}$ Just as with patients with tuberculosis not co-infected with HIV, they can also contribute to the onward transmission of Mycobacterium tuberculosis into the general population. ${ }^{3}$ Identification of HIV co-infection in people diagnosed with tuberculosis therefore enables more effective clinical management of both tuberculosis and HIV infection and provides the opportunity to reduce the risk of further transmission of either infection.

The proportion of adults in England and Wales with tuberculosis who were co-infected with HIV has been estimated previously. ${ }^{2-7}$ The results from these studies are difficult to compare because of differences in methods, settings, case definitions and time periods. In addition, migration trends have changed over the different time periods. In order to provide an up to date and systematic assessment of the impact of HIV infection on cases of active tuberculosis, we conducted a national population-based matching study which sought to estimate the proportion of HIV co-infection among cases of tuberculosis between 1999 and 2003 in England and Wales.
This paper describes recent trends in tuberculosis and HIV coinfection in population subgroups within England and Wales, and focuses on the implications of the findings for clinical and public health care.

\section{METHODS}

The Health Protection Agency Centre for Infections collates national surveillance data for reports of tuberculosis and HIV cases in separate databases. Cases of tuberculosis are primarily reported by respiratory and infectious disease clinicians, while HIV and AIDS diagnoses are supplied by physicians based in genitourinary and infectious disease clinics. Surveillance information at the Centre for Infections is collated through the "New diagnosis of HIV and AIDS" database for HIV infection, while tuberculosis surveillance data are collected through the Enhanced Tuberculosis Surveillance and the UK Mycobacterial Network (MycobNet) systems. Record linkage was used to match cases in the tuberculosis surveillance database (reported between 1999 and 2003) and the HIV surveillance database (reports from 1982 to 2003) to determine the prevalence of co-infection. It was not possible to match data in children aged $<15$ years because of a lack of unique identifiers. Cases of tuberculosis therefore included persons aged 15 years and over at diagnosis reported with active tuberculosis to the tuberculosis database and/or to the HIV surveillance database as an AIDS-defining illness between 1999 and 2003 in England and Wales. In-house matching software produced pairs of possible matches based on Soundex code, ${ }^{8}$ sex, residential address and date of birth. Pairs with a very high 
Table 1 Proportion of adults (aged $\geqslant 15$ years) with tuberculosis (TB) co-infected with HIV by demographic characteristics in England and Wales, 1999-2003

\begin{tabular}{|c|c|c|}
\hline Characteristic & $\begin{array}{l}\text { No (\% of total) } \\
\text { co-infected with } \\
\text { TB and HIV }\end{array}$ & Total with TB \\
\hline \multirow{2}{*}{\multicolumn{3}{|c|}{ Age group (years) }} \\
\hline & & \\
\hline $15-19$ & $11(0.6)$ & 1741 \\
\hline $20-29$ & $417(5.6)$ & 7482 \\
\hline $30-39$ & 853 (12.9) & 6639 \\
\hline $40-49$ & $336(8.1)$ & 4167 \\
\hline $50-64$ & $109(2.3)$ & 4733 \\
\hline $65+$ & $15(0.3)$ & 5772 \\
\hline Unknown & $2(1.5)$ & 136 \\
\hline \multicolumn{3}{|l|}{ Calendar year } \\
\hline 1999 & $169(3.1)$ & 5388 \\
\hline 2000 & $253(4.2)$ & 5990 \\
\hline 2001 & $314(5.1)$ & 6211 \\
\hline 2002 & $459(7.1)$ & 6497 \\
\hline 2003 & $548(8.3)$ & 6584 \\
\hline \multicolumn{3}{|l|}{ Sex } \\
\hline Male & $869(5.2)$ & 16788 \\
\hline Female & $845(6.1)$ & 13800 \\
\hline Unknown & $29(35.4)$ & 82 \\
\hline \multicolumn{3}{|l|}{ Ethnic group } \\
\hline White & $179(2.1)$ & 8668 \\
\hline Black African & $1217(20.5)$ & 5938 \\
\hline \multirow{2}{*}{\multicolumn{3}{|c|}{$\begin{array}{l}\text { Indan, Pakistani and } \\
\text { Bangladeshi }\end{array}$}} \\
\hline & & \\
\hline Mixed other & $58(2.5)$ & 2318 \\
\hline Black other & $16(7.8)$ & 204 \\
\hline Black Caribbean & $22(2.7)$ & 818 \\
\hline Unknown & $214(11.4)$ & 1871 \\
\hline \multicolumn{3}{|l|}{ Region of birth } \\
\hline Africa & $1166(19.8)$ & 5880 \\
\hline Asia & $57(0.6)$ & 9506 \\
\hline Europe & $48(4.6)$ & 1046 \\
\hline Latin America/Caribbean & 15 (3.5) & 430 \\
\hline North America/Australasia & $3(7.1)$ & 42 \\
\hline UK & $132(1.5)$ & 8646 \\
\hline Unknown & $322(6.3)$ & 5105 \\
\hline \multicolumn{3}{|l|}{ Born in UK } \\
\hline Yes & $132(1.5)$ & 8646 \\
\hline No & $1611(7.3)$ & 22024 \\
\hline \multicolumn{3}{|l|}{ Place of TB report } \\
\hline London & $903(6.9)$ & 13077 \\
\hline Rest of England \& Wales & $673(3.9)$ & 17431 \\
\hline \multicolumn{3}{|l|}{ Years since arrival in the UK } \\
\hline$\leqslant 5$ & $826(10.7)$ & 7704 \\
\hline$>5$ & $200(2.9)$ & 6798 \\
\hline Unknown & $585(7.8)$ & 7522 \\
\hline
\end{tabular}

323 cases of tuberculosis did not come from the TB surveillance database but were derived from HIV surveillance data.

degree of similarity were automatically matched. Further pairs with a high matching score were reviewed individually. Trends in tuberculosis/HIV co-infection were classified by year of tuberculosis diagnosis.

Table 2 Proportion of adults (aged $\geqslant 15$ years) with active tuberculosis (TB) reported only to the HIV surveillance database

\begin{tabular}{lcc}
\hline & $\begin{array}{l}\text { No }(\% \text { of total co-infected) not } \\
\text { reported as active cases of TB } \\
\text { to TB surveillance database }\end{array}$ & Total co-infected \\
\hline 1999 & $40(23.7)$ & 169 \\
2000 & $52(20.6)$ & 253 \\
2001 & $54(17.2)$ & 314 \\
2002 & $78(17.0)$ & 459 \\
2003 & $99(18.1)$ & 548 \\
Total & $323(18.5)$ & 1743 \\
\hline
\end{tabular}

The proportion of HIV co-infection among cases of tuberculosis was calculated using all eligible cases of tuberculosis (defined above) as the denominator and the sum of patients with tuberculosis who were matched to a HIV record and those with tuberculosis as an AIDS-defining illness in the HIV database as the numerator. The distribution and characteristics of the cases were examined and the proportion of the cases of tuberculosis co-infected with HIV each year was calculated to examine time trends. An extension of the Wilcoxon rank-sum test for trend across ordered groups was used to assess the statistical significance of the trend.

\section{RESULTS}

Of the 30670 patients reported with tuberculosis between 1999 and 2003 in England and Wales, 1743 (5.7\%) were identified as being co-infected with HIV (table 1). Of these 1743 individuals with tuberculosis and HIV co-infection, 323 (18.5\%) were reported only to the HIV/AIDS surveillance system (table 2).

\section{Demographic distribution of co-infection}

The majority of co-infected individuals were young adults (median age 34.7 years, interquartile range 30.1-40.3), black African (69.8\%), male (49.9\%) and born outside the UK $(75.7 \%)$. About one in ten $(10.3 \%)$ were of white ethnic group and only $37(2.1 \%)$ were of Indian, Pakistani and Bangladeshi origin. Most of the co-infected cases were reported in London $(57.3 \%)$. Among the foreign-born cases, and where the year of entry into the UK was available $(n=1026), 80.5 \%$ had arrived during the 5 years before the diagnosis of tuberculosis.

Cases of tuberculosis aged 30-39 years had the highest proportion of HIV co-infection (12.9\%), and also showed the greatest rise in the number of co-infected cases over the study period. Although there was a statistically significant difference in the proportion of co-infected cases between age groups $\left(\mathrm{p}<0.001, \chi^{2}\right.$ test $)$, there was no apparent trend.

The proportion of HIV co-infection among cases of tuberculosis varied across different ethnic groups by sex (table 3 ). Among cases of tuberculosis of black African ethnic origin, women $(24.0 \%)$ had a higher proportion co-infected with HIV than men $(17.1 \%)$ while, in the white ethnic group, the proportion co-infected with HIV was higher in men $(3.0 \%)$ than in women $(0.5 \%)$. Although more than one third $(35.4 \%)$ of all the cases of tuberculosis were from the Indian, Pakistani and Bangladeshi ethnic group, the proportion of these patients coinfected with HIV was much lower for both sexes (men $0.5 \%$, women $0.2 \%$ ) than in the other ethnic groups.

Of the patients with tuberculosis born outside the UK, 7.3\% were co-infected with HIV (table 1). Among this group of patients, those who had arrived in the UK during the 5 year period before their tuberculosis diagnosis had a higher proportion of HIV co-infection (10.7\%) than those who had arrived $>5$ years previously $(2.9 \%)$.

\section{Trends in proportion of co-infected cases}

Both the number of cases of tuberculosis and the proportion coinfected with HIV increased from 1999 to 2003. The proportion of patients co-infected with HIV increased each year from $3.1 \%$ $(169 / 5388)$ in 1999 to $8.3 \%(548 / 6584)$ in $2003(p<0.0001$, trend test, fig 1). Although the number of cases not known to the tuberculosis surveillance system increased from 40 in 1999 to 99 in 2003, there was no evidence of a significant trend $(\mathrm{p}=0.175$, trend test, table 2$)$.

The increase in proportion of co-infected cases over the study period was observed across all groups of patients regardless of their place of birth (fig 1). However, the percentage increase seen in patients born outside the UK (150\%) was more than twice that of patients born in the UK $(72.7 \%)$. This was despite 
Table 3 Proportion of adults (aged $\geqslant 15$ years) with tuberculosis (TB) co-infected with HIV by sex, ethnic group and place of birth reported in England and Wales, 1999-2003

\begin{tabular}{|c|c|c|c|c|c|c|c|}
\hline \multirow[b]{2}{*}{ Sex } & \multirow[b]{2}{*}{ Ethnic group } & \multicolumn{2}{|c|}{ UK born } & \multicolumn{2}{|c|}{ Non-UK born } & \multicolumn{2}{|l|}{ Total } \\
\hline & & TB & TB/HIV (\%)* & TB & TB/HIV (\%)* & TB & TB/HIV (\%)* \\
\hline \multirow[t]{8}{*}{ Men } & White & 4076 & $97(2.4)$ & 1262 & $65(5.2)$ & 5338 & $162(3.0)$ \\
\hline & Black African & 82 & $6(7.3)$ & 2922 & $508(17.4)$ & 3004 & $514(17.1)$ \\
\hline & Indian, Pakistani and Bangladeshi & 593 & $0(0.0)$ & 4884 & $28(0.6)$ & 5477 & $28(0.5)$ \\
\hline & Mixed/other & 46 & $2(4.4)$ & 1318 & $36(2.7)$ & 1364 & $38(2.8)$ \\
\hline & Black Caribbean & 222 & $2(0.9)$ & 280 & $13(4.6)$ & 502 & $15(3.0)$ \\
\hline & Black other & 23 & $0(0.0)$ & 103 & $12(11.7)$ & 126 & $12(9.5)$ \\
\hline & Unknown & 34 & $2(5.9)$ & 943 & $98(10.4)$ & 977 & $100(10.2)$ \\
\hline & Total & 5076 & $109(2.2)$ & 11712 & $760(6.5)$ & 16788 & $869(5.2)$ \\
\hline \multirow[t]{8}{*}{ Women } & White & 2566 & $11(0.4)$ & 763 & $6(0.8)$ & 3329 & $17(0.5)$ \\
\hline & Black African & 77 & $9(11.7)$ & 2846 & $693(24.4)$ & 2923 & $702(24.0)$ \\
\hline & Indian, Pakistani and Bangladeshi & 686 & $1(0.2)$ & 4667 & $8(0.2)$ & 5353 & $9(0.2)$ \\
\hline & Mixed/other & 55 & $0(0.0)$ & 895 & $20(2.2)$ & 950 & $20(2.1)$ \\
\hline & Black Caribbean & 150 & $2(1.3)$ & 166 & $5(3.0)$ & 316 & $7(2.2)$ \\
\hline & Black other & 12 & $0(0.0)$ & 66 & $4(6.1)$ & 78 & $4(5.1)$ \\
\hline & Unknown & 19 & $0(0.0)$ & 832 & $86(10.3)$ & 851 & $86(10.1)$ \\
\hline & Total & 3565 & $23(0.7)$ & 10235 & $822(8.0)$ & 13800 & $845(6.1)$ \\
\hline Unknown & & 5 & $0(0.0)$ & 77 & $29(37.7)$ & 82 & $29(35.4)$ \\
\hline Grand total & & 8646 & $132(1.5)$ & 22024 & $1611(7.3)$ & 30670 & $1743(5.7)$ \\
\hline
\end{tabular}

the slight decrease in the number of cases of tuberculosis seen in the latter group of patients over the study period.

An increase in the numbers of co-infected individuals was found across all ethnic groups. The highest percentage increase was observed among black Africans (100\%) followed by white patients $(78.6 \%)$ and those of Indian, Pakistani and Bangladeshi origin (50\%, table 4 ).

\section{Estimating the contribution of HIV co-infection to the national increase in tuberculosis}

Over the 5 year period, the number of reported cases of tuberculosis in persons aged 15-64 years in England and Wales increased by 1196 (from 5388 to 6584) and the number of known HIV co-infected cases increased by 379 (from 169 to 548). Thus, HIV infection is estimated to have contributed to a minimum $31.7 \%(379 / 1196)$ of the increase in cases of tuberculosis between 1999 and 2003 in this age group.

\section{DISCUSSION}

The number of individuals with tuberculosis and HIV Coinfection has increased significantly between 1999 and 2003 in

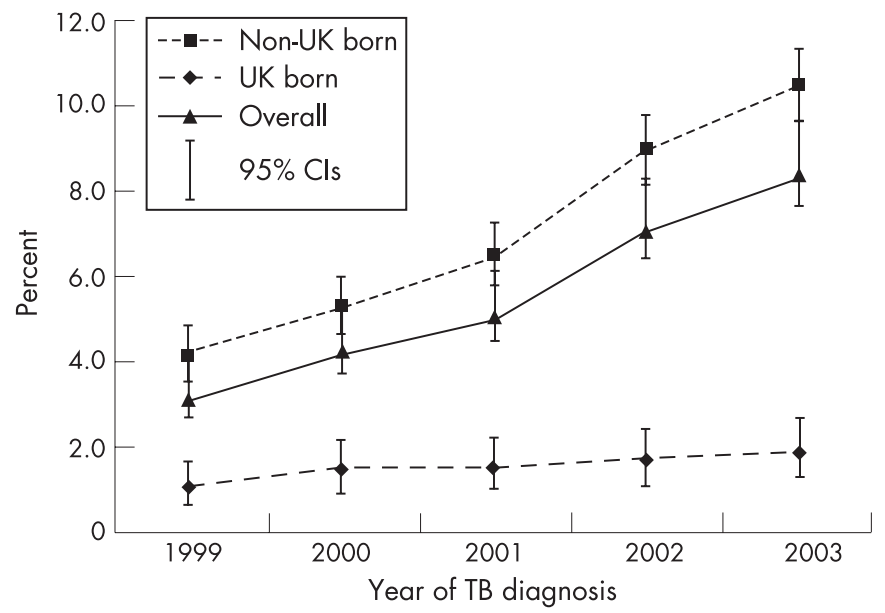

Figure 1 Proportion of adults (aged 15 years and over) with tuberculosis (TB) co-infected with HIV by place of birth and year of TB diagnosis reported in England and Wales, 1999-2003.
England and Wales. This is consistent with the overall trends in both conditions when taken separately. ${ }^{19}$ Over the study period about 1 in $17(5.7 \%)$ cases of tuberculosis were co-infected with HIV, with the highest estimate of 1 in $12(8.3 \%)$ observed in 2003. The proportion of patients with tuberculosis co-infected with HIV in this study is higher than previously reported estimates for England and Wales (4.6\% in 1993-4, ${ }^{7} 3.3 \%$ in $1998^{2}$ ) but less than those for the USA and Spain. ${ }^{10-12}$ As expected, a higher proportion of co-infected patients $(6.9 \%)$ was observed in London than in the rest of England and Wales $(3.9 \%)$. This finding falls within the previous range of estimates of $7 \%,{ }^{13} 11 \%{ }^{14}$ and $24.8 \%{ }^{6}$ reported from London.

HIV co-infection appears to be more common in patients with tuberculosis who are born outside the UK, particularly in those who have arrived in the country within the previous 5 years. A large proportion of cases were born in Africa or in a European country other than the UK. This is consistent with previous work, ${ }^{2}$ and mirrors the ethnic and gender mix of adults with HIV in England and Wales. ${ }^{15}$ The HIV epidemic across sub-Saharan Africa may partly explain the increase in the black African ethnic group. In contrast to a previous study ${ }^{2}$ that found no evidence of tuberculosis/HIV co-infection in certain ethnic groups such as Indians, Pakistanis and Bangladeshis between 1993 and 1998 in the UK, this study identified an increasing number of individuals with tuberculosis/HIV co-infection in all the ethnic groups examined. The contribution of the expanding HIV epidemic in the Indian subcontinent and Eastern Europe to the recent increases in coinfection rates needs further investigation.

The rising prevalence of undiagnosed HIV infection across the UK population and its impact on the incidence of tuberculosis is a cause for concern. Tuberculosis is now the most common AIDS-defining illness in the UK, accounting for $32 \%$ of all AIDS presentations in 2003. ${ }^{15}$

We estimated that patients co-infected with HIV have contributed to almost one third of the increase in the number of cases of tuberculosis in adults aged 15-64 years in England and Wales between 1999 and 2003. This is well above the proportion of 8.5\% reported between 1993 and 1998, ${ }^{2}$ suggesting a greater overlap between populations infected with HIV and those with tuberculosis.

As a population-based study that matched national surveillance databases, there are potential limitations to the findings. 
Table 4 Proportion of adults (aged $\geqslant 15$ years) with tuberculosis (TB) co-infected with HIV by ethnic group and year of TB diagnosis reported in England and Wales, 1999-2003

\begin{tabular}{|c|c|c|c|c|c|c|c|c|c|c|}
\hline \multirow[b]{2}{*}{ Ethnic group } & \multicolumn{2}{|l|}{1999} & \multicolumn{2}{|l|}{2000} & \multicolumn{2}{|l|}{2001} & \multicolumn{2}{|l|}{2002} & \multicolumn{2}{|l|}{2003} \\
\hline & TB & TB/HIV (\%) & TB & TB/HIV (\%) & TB & TB/HIV (\%) & TB & TB/HIV (\%) & TB & TB/HIV (\%) \\
\hline White & 1758 & $25(1.4)$ & 1845 & $37(2.0)$ & 1852 & $38(2.1)$ & 1633 & $39(2.4)$ & 1580 & $40(2.5)$ \\
\hline Black African & 833 & 105 (12.6) & 965 & $155(16.1)$ & 1041 & $208(19.9)$ & 1433 & $329(23.0)$ & 1666 & $420(25.2)$ \\
\hline $\begin{array}{l}\text { Indian, Pakistani and } \\
\text { Bangladeshi }\end{array}$ & 1998 & $4(0.2)$ & 2220 & $8(0.4)$ & 2133 & $6(0.3)$ & 2256 & $12(0.5)$ & 2246 & $7(0.3)$ \\
\hline Mixed/other & 314 & $6(1.9)$ & 379 & $9(2.4)$ & 456 & $11(2.4)$ & 596 & $19(3.2)$ & 574 & $13(2.3)$ \\
\hline Black Caribbean & 130 & $3(2.3)$ & 183 & $3(1.6)$ & 171 & $4(2.3)$ & 187 & $7(3.7)$ & 147 & $5(3.4)$ \\
\hline Black other & 21 & $0(0.0)$ & 26 & $0(0.0)$ & 32 & $2(6.3)$ & 53 & $10(18.9)$ & 72 & $4(5.6)$ \\
\hline Unknown & 334 & $36(7.8)$ & 372 & $41(11.0)$ & 526 & $45(8.6)$ & 340 & $43(12.7)$ & 299 & $59(19.7)$ \\
\hline Total & 5388 & $169(3.1)$ & 5990 & $253(4.2)$ & 6211 & $314(5.1)$ & 6497 & $459(7.1)$ & 6584 & $548(8.3)$ \\
\hline
\end{tabular}

The matching algorithms used to align the two surveillance databases may have missing codes and values. Both tuberculosis and HIV surveillance systems are subject to some underreporting, which limits the ability of this study to ascertain the true proportion of individuals co-infected with tuberculosis and HIV. While we cannot exclude the potential use of different names and dates of births from some population groups, the overall effect of these limitations will be to lead to an underestimate of the true proportion co-infected. A previous investigation by the Health Protection Agency concluded that different spellings of names, "difficult names" and multiple dates of birth did not significantly affect the overall picture of the HIV epidemic in the UK. ${ }^{16}$ The numbers presented in this report are therefore minimum estimates and the true extent of co-infection may in fact be larger.

Another limitation of this study is the inability of the surveillance system accurately to monitor multiple episodes of tuberculosis during the 5 year period. Nevertheless, this is unlikely to alter the results of this study as only $0.9 \%$ of cases reported a history of a previous diagnosis of tuberculosis in the study period.

\section{Public health implications}

The increased impact of HIV on tuberculosis demonstrates the importance of HIV prevention for effective tuberculosis control. The significant number of reported cases of tuberculosis, and the possibility of delay in disease progression among those with early diagnosed HIV infection, means that an increasing proportion of individuals may now present to non-genitourinary medicine physicians unaware of their concomitant HIV infection. This will remain a cause for concern within the context of the high prevalence of undiagnosed HIV infection in England and Wales. ${ }^{9}$

The National Institute for Clinical Excellence has recently published guidance on the management of tuberculosis, ${ }^{17}$ recommending that patients with tuberculosis should be assessed and those considered at risk of HIV infection offered testing. The continued increase in the proportion of cases of tuberculosis co-infected with HIV in England and Wales suggests that the recommendation by the British HIV Association $^{18}$ that all patients with tuberculosis should be offered HIV testing is reasonable. The routine offer of HIV testing helps provide an opportunity to promote and facilitate behaviour change among patients with tuberculosis and would enhance early access to appropriate clinical care and support to prevent onward transmission. ${ }^{19}$ A similar programme that offers confidential HIV testing to all pregnant women attending antenatal care regardless of their background has proved not only to be effective but also acceptable to patients. ${ }^{9}$ However, there may be practical challenges in offering testing within tuberculosis services, as highlighted in a recent report from a metropolitan centre. ${ }^{14}$

\section{Clinical implications}

About one in five of all co-infected patients were not reported as a case of active tuberculosis to the national tuberculosis surveillance system. It is possible that some of the individuals not reported to the national tuberculosis surveillance system were also not known to the local tuberculosis services. There may be a number of reasons for this, including failure of the matching process, patients moving to another clinic where it is assumed that they have already been notified and patients who disappear out of the system altogether once diagnosed with coinfection. It is also possible that some of these patients did not receive the recommended standard of clinical care for their tuberculosis, and that the appropriate public health measures around the case were not instituted. Equally, these cases may have been managed appropriately and just not reported. This issue warrants further study.

The British HIV Association recommends that tuberculosis/ HIV co-infection should be managed by clinicians with a specialist interest in both tuberculosis and HIV, ${ }^{18}$ and the British Thoracic Society recommends that all patients with tuberculosis should be supervised by physicians with full training in the management of tuberculosis. ${ }^{20}$ In practice, this can mean shared care which, in turn, requires a high degree of collaboration between the different clinical teams. A recent audit by the British HIV Association suggests that most tuberculosis and HIV clinicians are already working collaboratively in multidisciplinary teams, and that they routinely recommend HIV testing to all patients with newly diagnosed tuberculosis. A large proportion of these clinicians also reported prompt notification of all patients in their centre to their local consultant in communicable disease control. ${ }^{21}$

HIV infection is contributing significantly to the increase in tuberculosis in England and Wales. Our data may underestimate the size of this effect as it appears that a reasonable proportion of co-infected patients only encounter HIV services. Equally, non-HIV physicians who diagnose a case of tuberculosis need to be aware that HIV co-infection is present within all ethnic groups. HIV testing should be offered to adults with tuberculosis. Close cooperation in the management and reporting of these patients is recommended to ensure appropriate care and public health management.

\section{ACKNOWLEDGEMENTS}

The authors thank David Quinn and Tom Nichols for their contribution to the preparation of data for this study and acknowledge the support of all the health professionals who reported to the surveillance systems. 


\section{Authors' affiliations}

Aliko B Ahmed, Ibrahim Abubakar, Valerie Delpech, Delia Boccia, Josh Forde, Delphine Antoine, John M Watson, Centre for Infections, Health Protection Agency, Colindale, London, UK

Ibrahim Abubakar, School of Medicine, Health Policy and Practice,

University of East Anglia, Norwich, UK

Marc Lipman, Royal Free Hospital, London, UK

Funding: None.

Competing interests: None declared.

This study was carried out with national surveillance data. The Health Protection Agency has Patient Information Advisory Group approval to hold and analyse national surveillance data for public health purposes under Section 60 of the Health and Social Care Act 2001. No patients' names are collected for HIV surveillance; instead, surname Soundex codes are used and strict confidentiality of the data is maintained.

All authors have seen and approved the final manuscript. In addition, ABA wrote the first draft of the manuscript and IA conducted the analysis. All authors contributed to the design of the study, interpretation of the data and writing of the paper. JMW is guarantor.

\section{REFERENCES}

1 Health Protection Agency Centre for Infections. Annual report on tuberculosis cases reported in 2002 in England, Wales and Northern Ireland: enhanced tuberculosis surveillance. London: Health Protection Agency Centre for Infections, Tuberculosis Section, 2005.

2 Rose AM, Sinka K, Watson JM, et al. An estimate of the contribution of HIV infection to the recent rise in tuberculosis in England and Wales. Thorax 2002;57:442-5.

3 Lienhardt C, Rodrigues LC. Estimation of the impact of the human immunodeficiency virus infection on tuberculosis: tuberculosis risks re-visited? Int J Tuberc Lung Dis 1997;1:196-204.

4 Kumar D, Watson JM, Charlett A, et al. Tuberculosis in England and Wales in 1993: results of a national survey. Public Health Laboratory Service/British Thoracic Society/Department of Health Collaborative Group. Thorax 1997;52:1060-7
5 Bowen EF, Rice PS, Cooke NT, et al. HIV seroprevalence by anonymous testing in patients with Mycobacterium tuberculosis and in tuberculosis contacts. Lancet 2000;356:1488-9.

6 Marshall BG, Mitchell DM, Shaw RJ, et al. HIV and tuberculosis co-infection in an inner London hospital: a prospective anonymized seroprevalence study. J Infect 1999;38:162-6.

7 Watson JM, Meredith SK, Whitmore-Overton E, et al. Tuberculosis and HIV: estimates of the overlap in England and Wales. Thorax 1993;48:199-203.

8 Mortimer JY, Salathiel JA. 'Soundex' codes of surnames provide confidentiality and accuracy in a national HIV database. Commun Dis Rep CDR Rev 1995;5:R183-6.

9 UK Collaborative Group for HIV and STI Surveillance. Focus on prevention. HIV and other sexually transmitted infections in the United Kingdom in 2003. London: Health Protection Agency Centre for Infections, 2004.

10 Centers for Disease Control and Prevention. Reported tuberculosis in the United States, 1999. Atlanta: US Department of Health and Human Services, 2000.

11 Martin V, Cayla JA, Bolea A, et al. Mycobacterium tuberculosis and human immunodeficiency virus co-infection in intravenous drug users on admission to prison. Int J Tuberc Lung Dis 2000;4:41-6.

12 Smith DK, Gwinn M, Selik RM, et al. HIV/AIDS among African Americans: progress or progression? AIDS 2000;14:1237-48.

13 Hayward AC, Goss S, Drobniewski F, et al. The molecular epidemiology of tuberculosis in inner London. Epidemiol Infect 2002;128:175-84.

14 Dart S, Alder D, Mamdani M, et al. HIV testing in TB clinics: a problem in practice? Thorax 2006;61:271-72.

15 Health Protection Agency. HIV and AIDS in the UK in 2001: an update. London: Centre for Infections Health Protection Agency, HIV and STI Section, 2002.

16 Dougan S, Harris JP. Overcounting of black Africans in the UK: the problem of undetected record duplication. Commun Dis Public Health 2003;6:147-51.

17 National Collaborating Centre for Chronic Conditions. Tuberculosis: clinical diagnosis and management of tuberculosis, and measures for its prevention and control. London: Royal College of Physicians, 2006.

18 Pozniak AL, Miller RF, Lipman MC, et al. BHIVA treatment guidelines for tuberculosis (TB)/HIV infection 2005. HIV Med 2005;6(Suppl 2):62-83.

19 Voluntary HIV-1 Counseling and Testing Efficacy Study Group. Efficacy of voluntary HIV-1 counselling and testing in individuals and couples in Kenya, Tanzania, and Trinidad: a randomised trial, Lancet 2000;356:103-12.

20 Joint Tuberculosis Committee of the British Thoracic Society. Chemotherapy and management of tuberculosis in the United Kingdom: recommendations 1998. Thorax 1998;53:536-48.

21 British HIV Association (BHIVA). Managing tuberculosis co-infection. London: BHIVA Clinical Audit Report, 2005: 405.

\section{LUNG ALERT}

\section{Smoking cessation may improve the microbial flora profile}

A Brook I, Gober AE. Effect of smoking cessation on the microbial flora. Arch Otolaryngol Head Neck Surg. 2007;133:135-8.

he nasopharyngeal flora of smokers contains more potential pathogens and fewer bacteria with interfering capability than those of non-smokers. This paper studied the effect of smoking cessation on levels of these organisms. Two nasopharyngeal swabs were taken from 20 adults who had smoked at least 10 cigarettes a day for the previous 5 years. The first swab was taken while the patient was still smoking and the second 12-15 months after smoking cessation. None of the subjects had received antimicrobial treatment or had had a respiratory tract infection in the 3 months prior to testing.

Eleven potential pathogens were isolated from 9 of the 20 subjects before smoking cessation. Two potential pathogens were isolated from two individuals after smoking cessation $(\mathrm{p}<0.05)$. Bacterial interference was found in 35 instances against the four potential pathogens investigated (Streptococcus pneumoniae, Haemophilus influenzae, Moraxella catarrhalis and S pyogens) by 14 normal flora isolates before smoking cessation and in 116 instances by 42 isolates after smoking cessation $(\mathrm{p}<0.01)$.

This study confirms previous work by the authors, that the nasopharyngeal flora of smokers contains more potential pathogens and fewer aerobic and anaerobic bacteria with interfering capability than that of non-smokers. The authors also showed that the low levels of interfering bacteria revert to normal after smoking cessation. This is an important finding, as the presence of organisms with interfering potential may play a role in the prevention of upper respiratory tract infections, and this provides further evidence for the importance of smoking cessation.

S J Mehto

Senior House Officer, The Royal London Hospital, UK; sjmehta@doctors.org.uk 